\title{
COUNTEREXAMPLES CONCERNING POWERS OF SECTORIAL OPERATORS ON A HILBERT SPACE
}

\begin{abstract}
Arnaud Simard
We give explicit constructions of semigroups and operators with particular properties. First we build a bounded $C_{0}$-semigroup which is invertible and which is not similar to a semigroup of contractions. Afterwards we exhibit operators which admit bounded imaginary powers of angle $\omega>0$ on a Hilbert space but which do not admit a bounded functional calculus on the sector of angle $\omega$. (This gives the limit of McIntosh's fundamental result.) Finally we build, in the 2-dimensional Hilbert space, an operator which is not the negative generator of a semigroup of contractions, although its imaginary powers are bounded by $e^{\pi|s| / 2}$.
\end{abstract}

\section{INTRODUCTION AND RESULTS}

This article treats various problems about functional calculi associated to sectorial operators or to $C_{0}$-semigroups on Hilbert spaces. The first issue is a similarity problem for semigroups. We shall say that a semigroup $\left(T_{t}\right)_{t>0}$ on a Hilbert space $H$ is similar to a contraction semigroup if there exists an invertible operator $S$ in $B(H)$ (the algebra of all bounded operators on $H$ ) such that $\left\|S T_{t} S^{-1}\right\| \leqslant 1$ for all $t>0$. Historically, the problem we consider here begins with Foguel's example [3] of a power bounded operator $A$ which is not similar to any contraction, that is, there exists a constant $K>0$ such that $\left\|A^{n}\right\| \leqslant K$ for all $n \in \mathbb{N}$, but there is no invertible operator $S$ such that $\left\|S A S^{-1}\right\| \leqslant 1$. Much later, Le Merdy [6] exhibited an invertible power bounded operator not similar to any contraction. Considering a semigroup instead of a single operator, Packel [12] constructed a bounded $C_{0}$-semigroup $\left(T_{t}\right)_{t>0}$ which is not similar to any contraction semigroup. Logically the next step is to consider the case of an invertible bounded $C_{0}$-semigroup. In order to solve this problem we shall show:

THEOREM 1. Fix $\omega>0$. There exists a $C_{0}$-group $\left(T_{s}\right)_{s \in \mathbf{R}}$ on a Hilbert space $H$ such that:

(i) there is a constant $K>0$ such that $\left\|T_{s}\right\| \leqslant K e^{\omega|s|}$ for all $s \in \mathbb{R}$;

(ii) there is no invertible operator $S$ such that $\left\|S T_{s} S^{-1}\right\| \leqslant e^{\omega|s|}$ for all $s \in \mathbb{R}$.

Received 24th February, 1999

Copyright Clearance Centre, Inc. Serial-fee code: 0004-9727/99 \$A2.00+0.00. 
In particular $\left(e^{-\omega s} T_{s}\right)_{s>0}$ is a bounded invertible $C_{0}$-semigroup which is not similar to a semigroup of contractions.

This result answers a question of deLaubenfels (see [2, Open questions 2.6]). Note that we cannot expect to take $\omega=0$ in Theorem 1, indeed Nagy (see, for example, [14]) showed that every bounded group is similar to a group of contractions.

We now turn to the second result which is related to the bounded imaginary power property and functional calculus. We shall restrict our attention to Hilbert spaces though we could consider Banach spaces. We first recall a few standard definitions. Given $\theta \in(0, \pi)$, we let $\Sigma_{\theta}=\left\{z \in \mathbb{C}^{*}:|\arg z|<\theta\right\}$. Given an open set $\Omega \in \mathbb{C}$, we let $H^{\infty}(\Omega)$ be the Banach algebra of all bounded analytic functions $f: \Omega \rightarrow \mathbb{C}$ equipped with the supremum norm

$$
\|f\|_{H^{\infty}(\Omega)}=\sup \{|f(z)|: z \in \Omega\} .
$$

Given an operator $A$ on a complex Hilbert space $H$, we denote by $D(A)$ its domain, $N(A)$ its kernel, $R(A)$ its range, $\sigma(A)$ its spectrum and $\rho(A)$ its resolvent set, and by $R(\lambda, A)$ the operator $(\lambda-A)^{-1} \in B(H)$ for all $\lambda \in \rho(A)$.

Definition. Let $\omega \in(0, \pi)$ and let $A$ be an operator on a Hilbert space $H$. A sectorial operator of type $\omega$ is a closed, densely defined operator $A$ with dense range, such that $N(A)=\{0\}, \sigma(A) \subset \overline{\Sigma_{\omega}}$, and for all $\theta \in(\omega, \pi)$, there exists $C>0$ such that

$$
\left\|z(z-A)^{-1}\right\| \leqslant C \quad \forall z \in\left(\overline{\Sigma_{\theta}}\right)^{c} .
$$

If $A$ is a sectorial operator, we shall say that $A$ has bounded imaginary powers of angle $\theta$ if there exists a constant $M>0$ such that $\left\|A^{i s}\right\| \leqslant M e^{\theta|s|}$ for all $s \in \mathbb{R}$ (see [15] or [16] for details about the bounded imaginary power property).

Recall that we can always define a functional calculus for a sectorial operator. Indeed let $A$ be a sectorial operator of type $\omega$, for some $\omega \in(0, \pi)$, and let $\theta \geqslant \omega$. We denote by $\mathcal{R}_{\theta}$ the algebra generated by the constant function 1 and the rational functions of the form $z \mapsto(z-a)^{-1}$ where $a \notin \overline{\Sigma_{\theta}}$. Thus $\mathcal{R}_{\theta}$ is the algebra of all the rational functions $f$ with poles in $\mathbb{C} \backslash \overline{\Sigma_{\theta}}$ and with nonpositive degree. We equip this algebra with the norm given by the embedding of $\mathcal{R}_{\theta}$ into $H^{\infty}\left(\Sigma_{\theta}\right)$. We can then unambiguously define a homomorphism

$$
u_{\theta}: \mathcal{R}_{\theta} \rightarrow B(H) \quad u_{\theta}(f)=f(A) .
$$

We shall call this homomorphism the $\mathcal{R}_{\theta}$-functional calculus of $A$. In general, there is no reason why $u_{\theta}$ should be bounded. McIntosh [9] showed the equivalence between the bounded imaginary power property and bounded functional calculus on a Hilbert space. This result can be can rephrased as follows.

THEOREM 2. Let $A$ be a sectorial operator of type $\omega$ in a Hilbert space $H$. Then the following are equivalent:

(i) the map $u_{\theta}$ is bounded for all $\theta>\omega$; 
(ii) the map $u_{\theta}$ is bounded for some $\theta>\omega$;

(iii) $A$ has bounded imaginary powers of angle $\theta$ for all $\theta>\omega$;

(iv) $A$ has bounded imaginary powers of angle $\theta$ for some $\theta>\omega$.

We are interested in what happens when $\theta=\omega$. We shall prove the following result.

THEOREM 3. Let $A$ be a sectorial operator of type $\omega>0$ in a Hilbert space $H$. If $u_{\omega}$ is bounded, then $A$ has bounded imaginary powers of angle $\omega$. Conversely, there exists a sectorial operator of type $\omega>0$ in a Hilbert space $H$ which has bounded imaginary powers of angle $\omega$ for which $u_{\omega}$ is not bounded.

We note that this result also answers a question of deLaubenfels (see [2, Open questions 2.6]).

It is known that the notions of sectorial operators and functional calculus are closely related to semigroups. We follow the usual terminology and say that an operator $A$ is maximal accretive if $-A$ is the generator of a semigroup of contractions. If $A$ is maximal accretive on a Hilbert space $H$, then its Cayley transform $(A-I)(A+I)^{-1}$ is a contraction on $H$ (see, for example, [7]). Hence, from von Neumann's inequality, $A$ admits a contractive $\mathcal{R}_{\pi / 2}$-functional calculus and $\left\|A^{i s}\right\| \leqslant e^{\pi \mid s / / 2}$ for all $s \in \mathbb{R}$. The Hille-Yosida theorem implies that if $u_{\pi / 2}$ is contractive then $A$ is maximal accretive. Section 3 is devoted to proving that the condition that $\left\|A^{i s}\right\| \leqslant e^{\pi|s| / 2}$ for all $s \in \mathbb{R}$ is not sufficient for $A$ to be maximal accretive. Indeed, we shall prove the following more general result.

THEOREM 4. For all $\omega>0$ there exists a sectorial operator $A$ on the Hilbert space $\mathbb{C}^{2}$ such that $\left\|A^{i s}\right\| \leqslant e^{\omega|s|}$ for all $s \in \mathbb{R}$, and $u_{\omega}$ is not a contractive homomorphism.

In particular when $\omega=\pi / 2$ we obtain an example of a sectorial operator $A$ such that $\left\|A^{i s}\right\| \leqslant e^{\pi|s| / 2}$ for all $s \in \mathbb{R}$, but $-A$ does not generate a semigroup of contractions.

\section{SIMILARITY PROBLEMS}

In this section we shall prove Theorem 1 and Theorem 3 by explicit constructions, based on the relationship between interpolating sequences, conditional bases, and functional calculus.

We introduce notations and definitions. In this section, $\left(e_{n}\right)_{n \in \mathbb{N}}$ will be a Schauder basis of the Hilbert space $H$. That is, $\mathcal{V}=\operatorname{span}\left\{e_{n}: n \in \mathbb{N}\right\}$ is dense in $H$, and for all $x \in H$ there exists a unique sequence $\left(x_{n}\right)_{n \in \mathbf{N}} \subset \mathbb{C}$ such that $x=\sum_{n \in \mathbf{N}} x_{n} e_{n}$. In particular, this implies that there exists a constant $C>0$ such that

$$
\left\|\sum_{j=0}^{n} \alpha_{j} e_{j}\right\| \leqslant C\left\|\sum_{j=0}^{\infty} \alpha_{j} e_{j}\right\|
$$

for any finite sequence $\left(\alpha_{n}\right)_{n \in \mathbf{N}} \subset \mathbb{C}$ and any $n \geqslant 0$. Given a scalar sequence $\left(a_{n}\right)_{n \in \mathbf{N}}$, we define the diagonal linear map $M_{a}: \mathcal{V} \rightarrow \mathcal{V}$ by setting $M_{a}\left(e_{n}\right)=a_{n} e_{n}$ for all $n \in \mathbb{N}$. We 
set

$$
\left\|\left(a_{n}\right)_{n \in \mathbb{N}}\right\|=\limsup _{N \rightarrow+\infty}\left|a_{N}\right|+\sum_{N=0}^{+\infty}\left|a_{N+1}-a_{N}\right| ;
$$

then a standard Abel summation argument shows that if $\left\|\left(a_{n}\right)_{n \in \mathbb{N}}\right\|<\infty$ then $M_{a}$ is bounded and

$$
\left\|M_{a}\right\| \leqslant C\left\|\left(a_{n}\right)_{n \in \mathbb{N}}\right\| .
$$

If $\left(a_{n}\right)_{n \in \mathbb{N}}$ is a nondecreasing sequence of strictly positive real numbers then $M_{a}$ is closable and if we denote its closure by $A$, then $\sigma(A) \subset\left[a_{0},+\infty\right)$. Moreover $A$ is sectorial of any positive type (see [1] for details). Finally, we may easily check that if $f \in \mathcal{R}_{\theta}$, where $\theta>0$, then $f(A)$ is the closure of the map $M_{f(a)}$, which sends $e_{n}$ to $f\left(a_{n}\right) e_{n}$ for all $n \in \mathbb{N}$. We now turn to the definition of an interpolating sequence.

DEFINITION. Let $\Omega$ be an open subset of the complex plane. A scalar sequence $\left(\alpha_{n}\right)_{n \in \mathbb{N}} \subset \Omega$ is an interpolating sequence for $\Omega$ if and only if, given any bounded scalar sequence $\left(z_{n}\right)_{n \in \mathbb{N}}$, there exists a function $f \in H^{\infty}(\Omega)$ such that $f\left(\alpha_{n}\right)=z_{n}$ for all $n \in \mathbb{N}$ (see, for example, Garnett [4] for more about interpolating sequences).

2.1. Proof of Theorem 1: Fix $\omega>0$, and let $\left(e_{n}\right)_{n \in \mathbb{N}}$ be a conditional Schauder basis of the Hilbert space $H$, that is, there exists a sequence $\left(\varepsilon_{n}\right)_{n \in \mathbb{N}} \in\{-1,+1\}^{\mathbb{N}}$ such that $M_{\varepsilon}$ is unbounded.

Let $\left(a_{n}\right)_{n \in \mathbb{N}}$ be a nondecreasing sequence of positive real numbers such that $a_{0}>0$, $\lim _{n \rightarrow \infty} a_{n}=1$, and $\left(a_{n}\right)_{n \in \mathbb{N}}$ is an interpolating sequence for the open unit disk $D(0,1)$. Such a sequence exists (see [4]) and moreover we can choose any arbitrary value for $a_{0} \in(0,1)$; we shall choose $a_{0}>e^{-2 \omega}$. Let $A$ be the closure of the map $M_{a}$. From (2.1) and (2.2), we see that $\|A\| \leqslant 2 C$, and moreover $A$ is invertible and $A^{-1}$ is the closure of $M_{a^{-1}}$ so that we have $\left\|A^{-1}\right\| \leqslant C\left(1+a_{0}^{-1}\right)$. Since $A$ is bounded and invertible we can use the Dunford functional calculus to define

$$
A^{s}=\frac{1}{2 i \pi} \int_{\Gamma} \lambda^{s} R(\lambda, A) d \lambda
$$

for all $s \in \mathbb{R}$, where $\Gamma$ is a piecewise smooth simple closed path winding counterclockwise around $\sigma(A)$, which does not intersect the nonpositive real axis $(-\infty, 0]$. We deduce from (2.3) that $A^{s}$ coincides with $M_{a}$ on $\mathcal{V}$ for any $s \in \mathbb{R}$. Therefore, applying (2.1) and (2.2), we see that

$$
\begin{gathered}
\left\|A^{s}\right\| \leqslant C\left(2-a_{0}^{s}\right) \leqslant 2 C \quad \forall s \geqslant 0 . \\
\left\|A^{s}\right\| \leqslant C a_{0}^{s} \quad \forall s<0 .
\end{gathered}
$$

Since we chose $a_{0}>e^{-2 \omega}$,

$$
\left\|A^{s}\right\| \leqslant C e^{2 \omega|s|} \quad \forall s<0
$$


We shall now show that $A$ cannot be similar to any contraction.

If $A$ were similar to a contraction then from von Neumann's inequality we would obtain that

$$
\|f(A)\| \leqslant K \sup \{|f(z)|:|z| \leqslant 1\}
$$

for every function $f$ in the disk algebra $\mathcal{A}(\mathcal{D}(\prime, \infty))$. Now let $f$ be in $H^{\infty}(D(0,1))$, and let $f_{r}$ be its Poisson approximation, defined by $f_{r}(z)=f(r z)$ for any $z$ in $D(0,1)$ and $r<1$. Since $f_{r}$ belongs to $\mathcal{A}(\mathcal{D}(1, \infty)),(2.7)$ implies that $\left\|f_{r}(A)\right\| \leqslant K \sup \left\{\left|f_{r}(z)\right|:|z| \leqslant 1\right\}$. It is quite easy to check that $f_{r}(A)$ coincides with $M_{f_{r}(a)}$ on $\mathcal{V}$. And it is known that the norm of the diagonal map $M_{f_{r}(a)}$ may be computed using linear combinations, so we may let $r$ tend to 1 . Therefore

$$
\left\|M_{f(a)}\right\| \leqslant K\|f\|_{H^{\infty}(D(0,1))} \quad \forall f \in H^{\infty}(D(0,1)) .
$$

Since $\left(a_{n}\right)_{n \in \mathbb{N}}$ is an interpolating sequence, there exists $F \in H^{\infty}(D(0,1))$ such that $F\left(a_{n}\right)=\varepsilon_{n}$ for all $n \in \mathbb{N}$ where $\left(\varepsilon_{n}\right)_{n \in \mathbb{N}}$ is such that $M_{\varepsilon}$ is unbounded. Since $M_{F(a)}$ coincides with $M_{\varepsilon}$ on $\mathcal{V}$,

$$
M_{F(a)} \text { is unbounded. }
$$

This last statement (2.9) contradicts (2.8). Therefore $A$ is not similar to any contraction.

We now consider $T_{s}=e^{\omega s} A^{s}$. From (2.3), (2.4) and (2.6), it follows that $\left(T_{s}\right)_{s \in \mathbf{R}}$ is a uniformly continuous group which satisfies $\left\|T_{s}\right\| \leqslant K e^{\omega|s|}$ for all $s \in \mathbb{R}$, where $K=2 C$. Since $A$ is not similar to a contraction, there is no invertible operator $S$ such that $\left\|S A S^{-1}\right\| \leqslant 1$, hence there is no invertible operator $S$ such that $\left\|S T_{1} S^{-1}\right\| \leqslant e^{\omega}$. Therefore $\left(T_{s}\right)_{s \in \mathbf{R}}$ satisfies Theorem 1.

2.2. Proof of Theorem 3: Suppose first that $A$ is a sectorial operator of type $\omega>0$ in a Hilbert space $H$ and that $u_{\omega}$ is bounded.

For $\varepsilon \in(0,1)$, we consider the function $\rho_{\varepsilon}: z \in \Sigma_{\omega} \mapsto(\varepsilon+z) /(1+\varepsilon z)$. Then $\rho_{\varepsilon} \in \mathcal{R}_{\omega}$; we denote by $A_{\varepsilon}$ the operator $\rho_{\varepsilon}(A)$.

First we sketch the proof of the following lemma.

LEMma 5. Let $A$ be a sectorial operator of type $\omega>0$. If the $\mathcal{R}_{\omega}$-functional calculus $u_{\omega}$ of $A$ is bounded and $\left\|u_{\omega}\right\| \leqslant C$, then $\left\|f\left(A_{\varepsilon}\right)\right\| \leqslant C\|f\|$ for all $f \in H^{\infty}\left(\Sigma_{\omega}\right)$ and $\varepsilon \in(0,1)$.

Proof: We first fix $\varepsilon \in(0,1)$. Given $f \in H^{\infty}\left(\Sigma_{\omega}\right)$, we may find, by Runge's theorem, a sequence $\left(f_{n}\right) \subset \mathcal{R}_{\omega}$ such that

$$
\lim _{n} f_{n}\left(A_{\varepsilon}\right)=f\left(A_{\varepsilon}\right) \lim _{n}\left(\sup \left\{\left|\left(f-f_{n}\right)\left((\varepsilon+\lambda)(1+\varepsilon \lambda)^{-1}\right)\right|: \lambda \in \Sigma_{\omega}\right\}\right)=0 .
$$

Since $u_{\omega}$ is bounded and $f_{n} \circ \rho_{\varepsilon}$ belongs to $\mathcal{R}_{\omega}$,

$$
\left\|f_{n}\left(A_{\varepsilon}\right)\right\| \leqslant C \sup \left\{\left|f_{n} \circ \rho_{\varepsilon}(\lambda)\right|: \lambda \in \Sigma_{\omega}\right\} \quad \forall \varepsilon \in(0,1) .
$$


It follows from $(2.10)$ that $\left\|f\left(A_{\varepsilon}\right)\right\| \leqslant C\|f\|$.

To finish the proof of the first part of Theorem 3, we define the function $f_{s}$ in $H^{\infty}\left(\Sigma_{\omega}\right)$ for $s \in \mathbb{R}$, by $z \mapsto z^{i s}$. The lemma implies that

$$
\left\|f_{s}\left(A_{\varepsilon}\right)\right\|=\left\|A_{\varepsilon}^{i s}\right\| \leqslant C\left\|f_{s}\right\|_{H^{\infty}\left(\Sigma_{\omega}\right)} \leqslant C e^{\omega|s|}
$$

for all $\varepsilon \in(0,1)$. Now arguing as in Prüss $\left[15\right.$, Chapter 8], we conclude that $\left\|A^{i s}\right\| \leqslant C e^{\omega|s|}$ for all $s \in \mathbb{R}$, that is, $A$ has bounded imaginary powers of angle $\omega$. This ends the proof of the first part of Theorem 3 .

We now turn to the converse. We work in the same framework and with the same notation as in the proof of Theorem 1. We fix $\omega \in(0, \pi)$, and define $A$ as in the proof of Theorem 1. Since $A$ is bounded and invertible we may consider the operator $B=\left(e^{\omega} A\right)^{i}$ associated to the diagonal map $M_{b}$ where $b=\left(b_{n}\right)_{n \in \mathbb{N}}=\left(\left(e^{\omega} a_{n}\right)^{i}\right)_{n \in \mathbb{N}}$. It follows that $B^{i s}=\left(e^{\omega} A\right)^{-s}=T_{-s}$ for all $s \in \mathbb{R}$, hence $\left\|B^{i s}\right\| \leqslant K e^{\omega|s|}$.

Recall that $F$ is a function in $H^{\infty}(D(0,1))$ such that $M_{F(a)}$ is unbounded. Let us consider $k: \Sigma_{\omega} \rightarrow D\left(0, e^{\omega}\right)$, given by $z \mapsto z^{-i}$ and $h: D\left(0, e^{\omega}\right) \rightarrow D(0,1)$ given by $z \mapsto e^{-\omega} z$. Let $G=F \circ h \circ k$; clearly $G \in H^{\infty}\left(\Sigma_{\omega}\right)$. If the $\mathcal{R}_{\omega}$-functional calculus of $B$ were bounded, then it would follows from Lemma 1 that there exists a constant $C>0$ such that

$$
\left\|G\left(B_{\varepsilon}\right)\right\| \leqslant C\|G\|_{H^{\infty}\left(\Sigma_{\omega}\right)}
$$

for all $\varepsilon \in(0,1)$. Since $G\left(B_{\varepsilon}\right)$ coincides with the diagonal map $M_{G \circ \rho_{\varepsilon}(b)}$ on $\mathcal{V}$ we can let $\varepsilon$ tend to 0 to obtain

$$
\left\|M_{G(b)}\right\| \leqslant C\|G\|_{H^{\infty}\left(\Sigma_{w)}\right.}
$$

It suffices to observe that $M_{G(b)}$ coincides with $M_{F(a)}$ to contradict (2.9). Hence we conclude that $B$ cannot have a bounded $\mathcal{R}_{\omega}$-functional calculus.

\section{Problems of SEMigroups and THE Bounded imaginary PoWer property}

The main goal of this section is to prove Theorem 4 . We shall work on the Hilbert space $\mathbb{C}^{2}$. First we shall give some estimates for particular $2 \times 2$-matrices, and then we shall construct operators which satisfy Theorem 4 . We shall use this construction to prove a result concerning powers of sectorial operators and finally we shall give some further results arising, in part, from a result of Alan McIntosh.

3.1. Proof of Theorem 4: Let us consider $2 \times 2$-matrices of the form (from [13, Chapter 9] and [10])

$$
\left(\begin{array}{ll}
1 & z \\
0 & 1
\end{array}\right)
$$

where $z \in \mathbb{C}$. We shall use the estimate

$$
\left\|\left(\begin{array}{ll}
1 & z \\
0 & 1
\end{array}\right)\right\| \leqslant e^{|z| / 2} .
$$


We prove (3.1). It is easy to check that for any real $x>0$,

$$
\left\|\left(\begin{array}{ll}
1 & x \\
0 & 1
\end{array}\right)\right\|=\frac{x+\sqrt{x^{2}+4}}{2} .
$$

But $x>0$ implies that $\sinh x \geqslant x$ and then $\sinh x+\cosh x \geqslant x+\sqrt{x^{2}+1}$ so that $e^{x / 2} \geqslant\left(x+\sqrt{\left(x^{2}+4\right)}\right) / 2$. Hence

$$
\left\|\left(\begin{array}{ll}
1 & x \\
0 & 1
\end{array}\right)\right\| \leqslant e^{x / 2}
$$

Now we fix $z \in \mathbb{C}$ and denote $z=|z| e^{i \delta}$, with $\delta \in \mathbb{R}$.

$$
\left\|\left(\begin{array}{ll}
1 & z \\
0 & 1
\end{array}\right)\right\|=\left\|\left(\begin{array}{cc}
e^{i \delta} & 0 \\
0 & 1
\end{array}\right)\left(\begin{array}{cc}
1 & |z| \\
0 & 1
\end{array}\right)\left(\begin{array}{cc}
e^{-i \delta} & 0 \\
0 & 1
\end{array}\right)\right\|=\left\|\left(\begin{array}{cc}
1 & |z| \\
0 & 1
\end{array}\right)\right\| \leqslant e^{|z| / 2} .
$$

Clearly,

$$
f\left(\begin{array}{ll}
1 & z \\
0 & 1
\end{array}\right)=\left(\begin{array}{cc}
f(1) & z f^{\prime}(1) \\
0 & f(1)
\end{array}\right)
$$

for all $f \in H^{\infty}\left(\Sigma_{\omega}\right)$ and $\omega>0$. Fix $\beta \in \mathbb{R}_{+}$such that

$$
2 \sin \beta>\cosh \beta \text {. }
$$

(One can check that, for example, $\beta=\log 3$ satisfies (3.3)). Consider the operator $B=\left(\begin{array}{cc}1 & -2 i \sin \beta \\ 0 & 1\end{array}\right)$. The choice (3.3) implies that:

$$
v_{\beta}: f \in H^{\infty}\left(\Sigma_{\beta}\right) \mapsto f(B) \in B(H) \text { is not contractive. }
$$

Indeed, if we consider the function $f: z \mapsto z^{i}-z^{-i}$, then by (3.2) we have

$$
f(B)=\left(\begin{array}{cc}
0 & 4 \sin \beta \\
0 & 0
\end{array}\right)
$$

so that $\|f(B)\|=4 \sin \beta$. But

$$
\|f\|_{H^{\infty}\left(\Sigma_{\beta}\right)} \leqslant e^{\beta}+e^{-\beta}=2 \cosh \beta
$$

Since $2 \sin \beta>\cosh \beta$ we cannot have $\|f(B)\| \leqslant\|f\|_{H^{\infty}}\left(\Sigma_{\beta}\right)$.

Given $\omega \in(0, \pi)$ we choose $\zeta \in \mathbb{R}_{+}$such that $\omega=\zeta \beta$. Let us consider the operator $A$ defined by $A=B^{\varsigma}$. For all $s \in \mathbb{R},(3.2)$ and (3.1) imply

$$
\left\|A^{i s}\right\|=\left\|\left(\begin{array}{cc}
1 & 2 s \zeta \sin \beta \\
0 & 1
\end{array}\right)\right\| \leqslant e^{\zeta \sin \beta|s|} \leqslant e^{\zeta \beta|s|}=e^{\omega|s|},
$$

which gives (i). Finally (3.4) implies that the operator $f \mapsto f(A)$ on $H^{\infty}\left(\Sigma_{\omega}\right)$ cannot be contractive. Reasoning by approximation, as in the proof of Lemma 1, this implies that the $\mathcal{R}_{\omega}$-functional calculus associated to $A$ cannot be contractive. 
3.2. FURTHER RESULTS We shall use the construction above to construct a particular example concerning analytic semigroups. We recall that a semigroup $(T(t))_{t \geqslant 0}$ is said to be a bounded analytic semigroup if $t \mapsto T(t)$ admits a bounded analytic extension to some sector $\Sigma_{\theta}$ where $\theta>0$. In particular when $\left\|T_{z}\right\| \leqslant 1$ for all $z \in \Sigma_{\theta}$, we say that $\left(T_{z}\right)_{z \in \Sigma_{\theta}}$ is an analytic semigroup of contractions of angle $\theta$. For general background on analytic semigroups, we refer, for example, to [11].

Proposition 6 . There exists a sectorial operator $A$ on the Hilbert space $\mathbb{C}^{2}$ such that

(i) There exists $\alpha \in(0,1)$ such that $-A^{\alpha}$ generates an analytic semigroup of contractions of angle $\pi(1-\alpha) / 2$.

(ii) $-A$ does not generate a semigroup of contractions.

ProOF: In the construction above, we fix $\omega=\pi / 2$. We consider the operator $A=B^{\pi /(2 \beta)}$ and we take $\alpha=2 \beta / \pi$.

First we show that $-A^{\alpha}$ generates an analytic semigroup of contractions of angle $\pi / 2-\beta=\pi(1-\alpha) / 2$. We know that $-A^{\alpha}=-B$. We fix $s \in \mathbb{R}_{+}$and consider the entire function $f_{s}: z \mapsto e^{-s e^{i \beta^{\prime}} z}$ where $\beta^{\prime} \in[\beta-\pi / 2, \pi / 2-\beta]$. From (3.2),

$$
e^{-s e^{i \beta^{\prime} B}}=f_{s}(B)=e^{-s e^{i \beta^{\prime}}}\left(\begin{array}{cc}
1 & 2 i \sin \beta s e^{i \beta^{\prime}} \\
0 & 1
\end{array}\right) \text {. }
$$

Then (3.1) implies that $\left\|f_{s}(B)\right\| \leqslant e^{s\left(\sin \beta-\cos \beta^{\prime}\right)}$. Since $\beta^{\prime} \in[\beta-\pi / 2, \pi / 2-\beta]$, we have $\cos \beta^{\prime} \geqslant \sin \beta$. Hence $\left\|e^{-s e^{i \beta^{\prime}} B}\right\| \leqslant 1$ for all $s \in \mathbb{R}_{+}$and all $\beta^{\prime} \in[\beta-\pi / 2, \pi / 2-\beta]$, which gives (i). We recall that $A$ is constructed so that its $\mathcal{R}_{\pi / 2}$-functional calculus is not contractive, hence $-A$ cannot generate a semigroup of contractions, proving (ii).

After a first version of this paper was circulated, Alan McIntosh pointed out to me that, (working on an infinite dimensional Hilbert space) we can deduce from [8] and [5] the following extension of Proposition 1.

LEMma 7. For all $\alpha \in(0,1)$ there exists a sectorial operator $B$ on a Hilbert space H such that

(i) $-B^{\alpha}$ generates an analytic semigroup of contractions of angle $\pi(1-\alpha) / 2$;

(ii) $B$ is not maximal accretive.

Proof: Given $\alpha \in(0,1)$, McIntosh [8] exhibited a sectorial operator $C$ of angle $\pi \alpha / 2$ on an infinite-dimensional Hilbert space $H$ such that $-C$ generates an analytic semigroup of contractions of angle $\pi(1-\alpha) / 2$ and such that the norms defined by $\left\|C^{1 / 2} u\right\|$ and $\left\|C^{* 1 / 2} u\right\|$ for all $u \in D\left(C^{1 / 2}\right) \cap D\left(C^{* 1 / 2}\right)$ are not equivalent. We consider the operator $B=C^{1 / a}$. It is clear that $B$ satisfies (i). If we assume that $B$ is maximal accretive then, from a result of Kato [5], the norms defined by $\left\|B^{\alpha / 2} u\right\|$ and $\left\|B^{* \alpha / 2} u\right\|$ are equivalent. Since $B^{\alpha / 2}=C^{1 / 2}$ and $B^{* \alpha / 2}=C^{* 1 / 2}$, the latter assertion contradicts the choice of the operator $C$. Hence this gives (ii). 
In the previous section we gave a constructive proof of Theorem 4 in the Hilbert space $\mathbb{C}^{2}$. We now give a shorter proof of the same result with $\mathbb{C}^{2}$ replaced by any infinite dimensional Hilbert space.

We consider an operator $B$ satisfying Lemma 2 for some $\alpha \in(0,1)$ on a Hilbert space $H$. Since $-B^{\alpha}$ generates an analytic semigroup of contractions of angle $\pi(1-\alpha) / 2$, we can easily show that $\left\|\left(B^{\alpha}\right)^{i s}\right\| \leqslant e^{\pi \alpha|s| / 2}$ for all $s \in \mathbb{R}$. Hence, if we set $A=B^{2 \omega / \pi}$, we have $\left\|A^{i s}\right\| \leqslant e^{\omega|s|}$ for all $s \in \mathbb{R}$. Thus the first condition of Theorem 4 is satisfied.

Now, since $B$ is not maximal accretive, its $\mathcal{R}_{\pi / 2}$ functional calculus is not contractive, and hence $A$ cannot admit a contractive $\mathcal{R}_{\omega}$ functional calculus. This proves the second point of Theorem 4 .

\section{REFERENCES}

[1] J.B. Baillon and P. Clement, 'Examples of unbounded imaginary powers of operators', J. Funct. Anal. 100 (1991), 419-434.

[2] R. deLaubenfels, 'Strongly continuous groups, similarity and numerical range on a Hilbert space', Taiwanese J. Math. 1, 127-133.

[3] S.R. Foguel, 'A counterexample to a problem of Sz-Nagy', Proc. Amer. Math. Soc. 15 (1964), 788-790.

[4] J.B. Garnett, Bounded analytic functions (Academic Press, New York, London, 1981).

[5] T. Kato, 'Fractional powers of dissipative operators', J. Math. Soc. Japan 13 (1961), 246-274.

[6] C. Le Merdy, 'The similarity problem for bounded analytic semigroups on Hilbert space', Semigroup Forum 56 (1998), 205-224.

[7] B. Sz-Nagy and C. Foias, Harmonic analysis of operators on Hilbert space (Akademiai Kiadó, Budapest, 1970).

[8] A. McIntosh, 'On the comparability of $A^{\frac{1}{2}}$ and $A^{* \frac{1}{2}}$, , Proc. Amer. Math. Soc. 32 (1972), 430-434.

[9] A. McIntosh, 'Operators which have an $H^{\infty}$-Functional calculus', in Miniconference on Operator Theory and Partial Differential Equations, Proc. Cent. Math. Analysis 13 (A.N.U., Canberra, 1986), pp. 210-231.

[10] G. Misra, 'Curvature inequalities and extremal properties of bundle shifts', J. Operator Theory 11 (1984), 305-317.

[11] A. Pazy, Semigroups of linear operators and applications to partial differential equations, Applied Math. Sci. 44 (Springer-Verlag, Berlin, Heidelberg, New York, 1983).

[12] E.W. Packel, 'A semigroup analogue of Foguel's counterexample', Proc. Amer. Math. Soc. 21 (1969), 240-244.

[13] V.I. Paulsen, Completely bounded maps and dilations, Pitman Research Notes in Math. 146 (Longman, Wiley, New York, 1986).

[14] G. Pisier, Similarity problems and completely bounded maps, Lecture Notes in Math. 1618 (Springer-Verlag, Berlin, Heidelberg, New York, 1995).

[15] J. Prüss, Evolutionary integral equations and applications (Birkhäuser-Verlag, Basel, 1993). 
[16] J. Prüss and H. Sohr, 'On operators with bounded imaginary powers in Banach spaces', Math. Z. 203 (1990), 429-452.

Equipe de Mathématiques de Basançon Université de Franche-Comté 25030 Besancon cedex

France

e-mail: simard@math.univ-fcomte.fr 